\title{
Displacive structural phase transitions and the magnetic ground state of quadruple perovskite $\mathrm{YMn}_{7} \mathrm{O}_{12}$
}

\author{
R. D. Johnson, ${ }^{1, *}$ D. D. Khalyavin, ${ }^{2}$ P. Manuel, ${ }^{2}$ Y. Katsuya,${ }^{3}$ M. \\ Tanaka, ${ }^{3}$ Y. Matsushita, ${ }^{4}$ L. Zhang, ${ }^{5,6}$ K. Yamaura, ${ }^{5,6}$ and A. A. Belik ${ }^{5}$ \\ ${ }^{1}$ Clarendon Laboratory, Department of Physics, University of Oxford, Oxford, OX1 3PU, United Kingdom \\ ${ }^{2}$ ISIS facility, Rutherford Appleton Laboratory-STFC, Chilton, Didcot, OX11 0QX, United Kingdom \\ ${ }^{3}$ Synchrotron X-ray Station at SPring-8, NIMS, Kouto 1-1-1, Sayo-cho, Hyogo 679-5148, Japan \\ ${ }^{4}$ National Institute for Materials Science (NIMS), Sengen 1-2-1, Tsukuba, Ibaraki 305-0047, Japan \\ ${ }^{5}$ Research Center for Functional Materials, National Institute for \\ Materials Science (NIMS), Namiki 1-1, Tsukuba, Ibaraki 305-0044, Japan \\ ${ }^{6}$ Graduate School of Chemical Sciences and Engineering, Hokkaido University, \\ North 10 West 8, Kita-ku, Sapporo, Hokkaido 060-0810, Japan
}

(Dated: December 12, 2018)

\begin{abstract}
We present an experimental study of the structural and magnetic phase transitions of the quadruple perovskite $\mathrm{YMn}_{7} \mathrm{O}_{12}$. On cooling below $640 \mathrm{~K}$ a displacive structural phase transition occurs, lowering the crystal symmetry from cubic $(\operatorname{Im} \overline{3})$ to monoclinic $(I 2 / m)$, driven by long range B site $\mathrm{Mn}^{3+}$ orbital order. A second, unconventional structural phase transition takes place on further cooling below $200 \mathrm{~K}$, which is characterised by static atomic displacements $\left(P 2_{1} / n\right)$ as well as a dipolar-glass-like state of yttrium. This low temperature structural transition is driven by the underbonding of yttrium, which is present due to the rigid framework of octahedra tilts unique to the quadruple perovskites that primarily accommodates A site manganese ions. A ground state ferrimagnetic structure was also determined, similar to that found in other $R \mathrm{Mn}_{7} \mathrm{O}_{12}$ compounds that do not present a low temperature structural phase transition.
\end{abstract}

\section{INTRODUCTION}

The perovskite crystal structure with chemical formula $\mathrm{ABX}_{3}$ is ubiquitous in condensed matter physics and inorganic chemistry owing primarily to its chemical versatility ${ }^{1}$. The rare-earth manganites form a subgroup of the perovskite-type phases in which $\mathrm{A}=$ rareearth, yttrium, or manganese, $\mathrm{B}=$ manganese, and $\mathrm{X}=$ oxygen. Even within this compositionally limited family a vast range of compounds have been synthesised that support technologically important materials properties such as multiferroicity ${ }^{2,3}$ and colossal magnetoresistance $^{4}$. The manganite perovskites are also canonical examples of crystalline systems that host fundamental and cross-coupled lattice, charge, orbital and spin degrees of freedom ${ }^{5-8}$.

The chemical flexibility of the rare-earth manganites is primarily rooted in a variety of octahedra tilt patterns through which rigid $\mathrm{MnO}_{6}$ units coherently rotate to accommodate $\mathrm{A}$ site cations with different ionic radii and bonding geometries ${ }^{9}$. The simple perovskites (chemical formula $R \mathrm{MnO}_{3}, R=$ rare earth or Y), have been synthesised for all $R$ from La to Lu, however those with smaller rare-earth radius ( $R=$ Dy-Lu, and Y) can only be stabilised under high pressure synthesis conditions ${ }^{10}$ (in the latter cases a hexagonal modification of the perovskite is stable under ambient growth conditions). For all $R \mathrm{MnO}_{3}$ perovskites, the rare earth ions are accommodated by varying degrees of an $a^{-} b^{+} a^{-}$octahedra tilt pattern (in Glazer notation) - the so-called $\mathrm{GdFeO}_{3}$ distortion ${ }^{11}$, such that their crystal structure adopts the orthorhombic Pnma space group directly from the melt.

The rare-earth quadruple perovskite manganites have formula $\left(R \mathrm{Mn}_{3}\right) \mathrm{Mn}_{4} \mathrm{O}_{12}$, and a large $a^{+} a^{+} a^{+}$octahedra tilt pattern (cubic space group $\operatorname{Im} \overline{3}$ ) accommodates a 1:3 chemical ordering of $R$ and $\mathrm{Mn}$ ions on the perovskite A sites, respectively ${ }^{12,13}$. In this case, the $\mathrm{MnO}_{6}$ tilts are primarily driven by the establishment of a square planar oxygen coordination of the majority A site Mn ions, giving them a strict valence of +3 . As a result, less flexibility is afforded to the bonding of the rare-earth ions or yttrium at the minority A site, especially when compared to the $\mathrm{GdFeO}_{3}$ distortion of the simple perovskites.

In this paper we report the $\operatorname{Im} \overline{3}$ high temperature crystal structure of the quadruple perovskite manganite $\mathrm{YMn}_{7} \mathrm{O}_{12}$. We show that at $T_{\mathrm{OO}}=640 \mathrm{~K}$, a displacive structural phase transition occurs at which the cooperative Jahn-Teller effect introduces $d_{3 z^{2}-r^{2}}$ orbital order on the octahedrally coordinated $\mathrm{Mn}^{3+} \mathrm{B}$ site cations, and the respective atomic displacements lower the crystal symmetry to monoclinic (space group $I 2 / m$ ), as previously reported at room temperature ${ }^{14}$. An isostructural phase transition, which has not been found in any other $R \mathrm{Mn}_{7} \mathrm{O}_{12}$ compounds, was also reported at $T_{\mathrm{s}} \sim 200 \mathrm{~K}$. Below $T_{\mathrm{s}}$, a negative thermal expansion of the lattice occured, as well as an anomalous increase in the yttrium isotropic atomic displacement parameter ${ }^{14}$. Through analysis of neutron powder diffraction data we have demonstrated that this phase transition is not isostructural. Instead, we have found that the $a^{+} a^{+} a^{+}$ octahedra tilts of $\mathrm{YMn}_{7} \mathrm{O}_{12}$ are insufficient to establish full bonding of the yttrium ions, and this underbonding drives a displacive phase transition at $200 \mathrm{~K}$. Remarkably, the transition is characterised by two symmetry breaking order parameters; one associated with a static displacement of oxygen atoms, and the other likely re- 
lated to a dipolar-glass-like state of yttrium ion displacements consistent with the observed negative thermal expansion. Both orders dramatically improve the bonding conditions of yttrium and could independently result in such a phase transition in other $R \mathrm{Mn}_{7} \mathrm{O}_{12}$ compounds with small rare-earth radii (e.g. Ho or $\mathrm{Er}^{15}$ ). Finally, we present the ground state ferrimagnetic structure of $\mathrm{YMn}_{7} \mathrm{O}_{12}$ which was found to be the same as that of other $R \mathrm{Mn}_{7} \mathrm{O}_{12}$ compounds ${ }^{16}$, and hence unmodified by the low temperature structural phase transition.

The manuscript is organised as follows. In Sec. II we describe sample synthesis and all experimental methods employed, in Sec. III B and III C we describe the 640 $\mathrm{K}$ and the $200 \mathrm{~K}$ displacive structural phase transitions, respectively, and in Sec. IIID we present the determination of the ground state magnetic structure. Finally, we summarise our conclusions in Sec. IV.

\section{EXPERIMENT}

Polycrystalline samples with nominal composition $\mathrm{Y}_{0.9} \mathrm{Mn}_{7} \mathrm{O}_{11.85}$ (hereafter referred to as $\mathrm{YMn}_{7} \mathrm{O}_{12}$ ) were prepared from stoichiometric mixtures of $\mathrm{Mn}_{2} \mathrm{O}_{3}$ and $\mathrm{Y}_{2} \mathrm{O}_{3}(99.9 \%)$. Single-phase $\mathrm{Mn}_{2} \mathrm{O}_{3}$ was prepared from commercial $\mathrm{MnO}_{2}(99.99 \%)$ by heating in air at $923 \mathrm{~K}$ for $24 \mathrm{~h}$. The mixtures were placed in $\mathrm{Au}$ or $\mathrm{Pt}$ capsules and treated at $6 \mathrm{GPa}$ and about $1570-1670 \mathrm{~K}$ for $2 \mathrm{~h}$ (heating time to the synthesis temperature was $10 \mathrm{~min}$ ) in a belt-type high-pressure apparatus. After the heat treatments, the samples were quenched to room temperature, and the pressure was slowly released. All obtained samples were black pellets. We emphasize that a sample with nominal composition $\mathrm{YMn}_{7} \mathrm{O}_{12}$ contained a large amount of $\mathrm{YMn}_{3} \mathrm{O}_{6}$ impurity (approximately 12 wt.\%). Shifting the nominal composition to $\mathrm{Y}_{0.9} \mathrm{Mn}_{7} \mathrm{O}_{11.85}$ was found to significantly reduce the impurity content.

Differential scanning calorimetry curves were recorded on a Mettler Toledo DSC1 STAR ${ }^{\mathrm{e}}$ system at a heating/cooling rate of $10 \mathrm{~K} / \mathrm{min}$ under $\mathrm{N}_{2}$ flow between 128 and $698 \mathrm{~K}$ in open $\mathrm{Al}$ capsules.

Low-temperature $(10 \mathrm{~K}$ to $300 \mathrm{~K})$ and hightemperature ( $298 \mathrm{~K}$ to $660 \mathrm{~K})$ x-ray powder diffraction data were measured on a RIGAKU SmartLab instrument using $\mathrm{CuK}_{\alpha 1}$ radiation, in the range $5^{\circ}<2 \theta<120^{\circ}$, with a step size of $0.01^{\circ}$, using a cryostat system and a furnace attachment, respectively. The diffraction data were analysed by the Rietveld method using RIETAN-2000 ${ }^{17}$.

Variable temperature magnetisation measurements were performed in a 100 Oe applied magnetic field on a dense pellet using a SQUID magnetometer (Quantum Design MPMS). The specific heat, $c_{p}$, was recorded on cooling from $300 \mathrm{~K}$ to $2 \mathrm{~K}$ in zero applied magnetic field by a pulse relaxation method using a commercial calorimeter (Quantum Design PPMS).

Synchrotron x-ray powder diffraction data were measured at 113,295 , and about $730 \mathrm{~K}$ on a large DebyeScherrer camera at the undulator beamline BL15XU of
SPring- $8^{18,19}$ The intensity data were collected between $3^{\circ}$ and $70.43^{\circ}$ at $0.003^{\circ}$ intervals in $2 \theta$; the incident beam was monochromated at $\lambda=0.65298 \AA$. The sample was packed into a Lindemann glass capillary (inner diameter: $0.1 \mathrm{~mm}$ ), which was rotated during the measurements.

Neutron powder diffraction measurements were performed on the WISH time-of-flight diffractometer ${ }^{20}$ at ISIS, the UK Neutron and Muon Spallation Source. The sample $(\sim 1.2 \mathrm{~g})$ was loaded into a cylindrical vanadium can and mounted within a ${ }^{4} \mathrm{He}$ cryostat. Data were collected with high counting statistics at a fixed temperature within each magnetic phase, and in the paramagnetic phase above and below the structural phase transition. Data were also collected with lower counting statistics on warming in the temperature range $1.5 \mathrm{~K}$ to $230 \mathrm{~K}$, having cooled the sample at $\sim 10 \mathrm{~K} / \mathrm{min}$. Neutron and synchrotron diffraction data were refined using Fullprof ${ }^{21}$.

\section{RESULTS AND DISCUSSION}

\section{A. Structural phase transitions}

Differential scanning calorimetry (DSC) measurements of $\mathrm{YMn}_{7} \mathrm{O}_{12}$ (Figure 1a) demonstrated two structural phase transitions at $T_{\mathrm{OO}}=640 \mathrm{~K}$ and $T_{\mathrm{s}}=200 \mathrm{~K}$ (no magnetic anomalies occured at these temperatures). Thermal hysteresis was observed at $T_{\mathrm{OO}}$ indicating that this transition is first order, whilst the DSC data were consistent with a second order phase transition at $T_{\mathrm{S}}$. Crystallographic lattice parameters were refined against laboratory based x-ray powder diffraction data as a function of tempertaure, and their values are plotted in Figures $1 \mathrm{~b}-\mathrm{d}$. The diffraction data showed that on cooling below $T_{\text {OO }}$ the lattice symmetry transitions from cubic to monoclinic. Anomalous changes in the monoclinic lattice parameters, including negative thermal expansion (Figure 1e), were observed at $T_{\mathrm{s}}$, but there was no evidence for further symmetry breaking - fully consistent with the diffraction data presented in reference 14 . The monoclinic crystal symmetry was then found to persist down to the lowest measured temperatures.

\section{B. High temperature cubic phase and the first structural phase transition}

The high temperature cubic phase of $\mathrm{YMn}_{7} \mathrm{O}_{12}$ was modeled by the $\operatorname{Im} \overline{3}$ crystal structure adopted by many quadruple perovksite manganites ${ }^{13}$, and refined against synchrotron x-ray powder diffraction data measured at $\sim 730 \mathrm{~K}$ (Figure 2). The model was found to be in excellent agreement with the data $(R=1.39 \%, w R=1.87 \%$, and $\left.R_{\text {Bragg }}=4.47 \%\right)$. The cubic lattice parameter refined to $7.43651(1) \AA$, and the atomic fractional coordinates and isotropic atomic displacement parameters are 


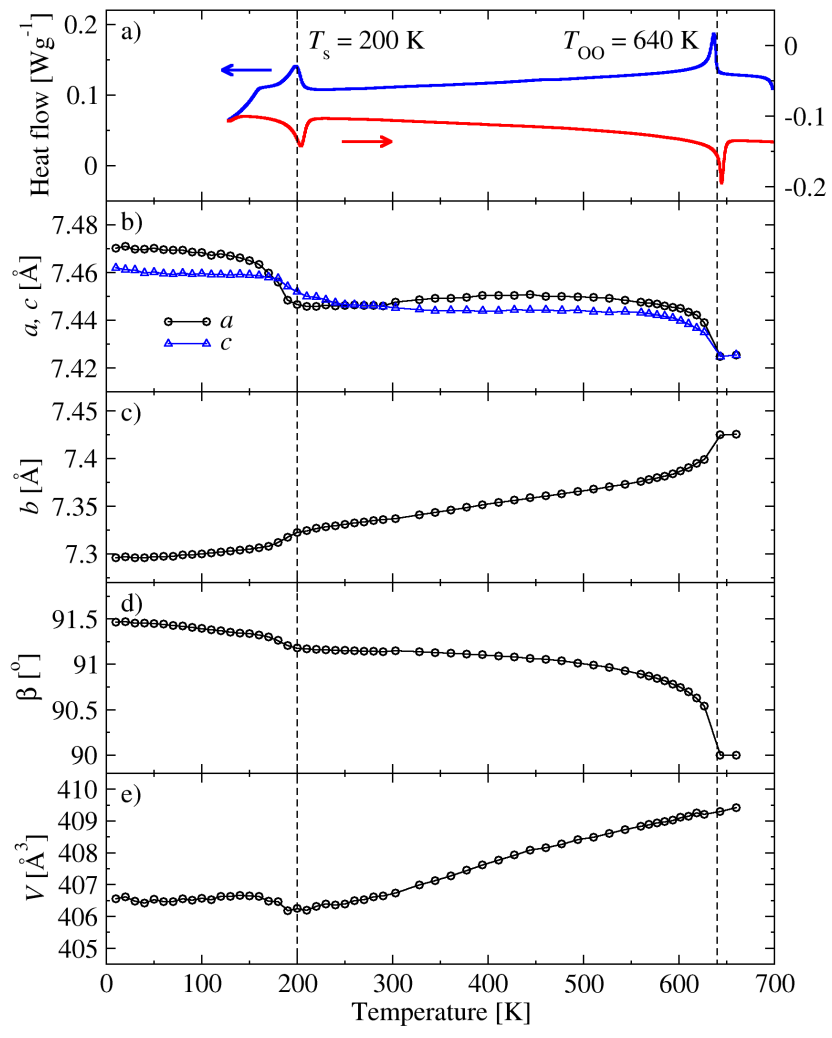

FIG. 1. The temperature dependence of a) differential scanning calorimetry curves measured on cooling (blue line) and warming (red line), (b-d) the lattice parameters, and (e) the unit cell volume.

TABLE I. Crystal structure parameters of $\mathrm{YMn}_{7} \mathrm{O}_{12}(Z=2)$ in the high temperature $\operatorname{Im} \overline{3}$ cubic phase $(\sim 730 \mathrm{~K})$. The cubic lattice parameter $a=7.43651(1) \AA$. Excellent reliability parameters of $R=1.39 \%, w R=1.87 \%$, and $R_{\mathrm{Bragg}}=4.47 \%$ were achieved in the refinement.

\begin{tabular}{c|ccccc}
\hline \hline Atom & Site & $x$ & $y$ & $z$ & $U_{\text {iso }}\left[\AA^{2}\right]$ \\
\hline $\mathrm{Y}$ & $2 a$ & 0 & 0 & 0 & $0.0235(7)$ \\
$\mathrm{Mn} 1$ & $6 b$ & 0 & $1 / 2$ & $1 / 2$ & $0.0207(5)$ \\
$\mathrm{Mn} 2$ & $8 c$ & $1 / 4$ & $1 / 4$ & $1 / 4$ & $0.0153(4)$ \\
$\mathrm{O}$ & $24 g$ & 0 & $0.3048(2)$ & $0.1693(3)$ & $0.0233(6)$ \\
\hline \hline
\end{tabular}

given in Table I. We note that a number of weak diffraction peaks were also present in the synchrotron x-ray powder diffraction data, which were not accounted for by the $\operatorname{Im} \overline{3}$ structural model. These peaks could be fit by a $2.58(5)$ wt\% $\mathrm{YMn}_{3} \mathrm{O}_{6}$ impurity phase ${ }^{15}$.

The room temperature crystal structure of $\mathrm{YMn}_{7} \mathrm{O}_{12}$ was reported to be monoclinic (space group $I 2 / m)^{14}$, which is consistent with our variable temperature powder x-ray diffraction data shown above (Figure 1), and confirmed by our neutron powder diffraction experiments discussed in Section III C. A modal decomposition of the structural distortions occuring below $T_{\text {OO }}$ was performed

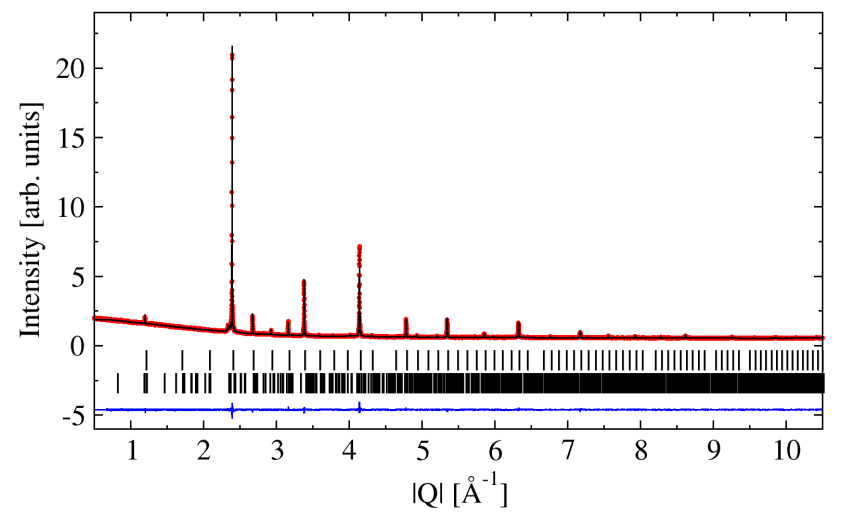

FIG. 2. Synchrotron x-ray powder diffraction data (red points) measured at $\sim 730 \mathrm{~K}$. The fitted $\mathrm{YMn}_{7} \mathrm{O}_{12}$ (top tick marks), and $\mathrm{YMn}_{3} \mathrm{O}_{6}$ impurity (bottom tick marks) structural models are shown as a solid black line. A difference pattern $\left(I_{\mathrm{obs}}-I_{\text {calc }}\right)$ is given as a solid blue line at the bottom of the figure.

using the ISOTROPY suite ${ }^{22,23}$. Taking the above $\operatorname{Im} \overline{3} \mathrm{cu}-$ bic structure as the parent, and the room temperature $I 2 / m$ phase reported in reference 14 as the distorted structure, we found that the primary displacive modes transform according to the $\Gamma_{4}^{+}$irreducible representation (Miller and Love notation). These atomic displacements accommodate a cooperative Jahn-Teller distortion of the $\mathrm{Mn}^{3+} \mathrm{O}_{6}$ B site octahedra - the so called $Q_{2}$ mode where the octahedral elongation axis lies approximately within the monoclinic ac-plane and alternates from one site to the next. Hence, long range $\mathrm{B}$ site $d_{3 z^{2}-r^{2}}$ orbital order was found to drive the cubic to monoclinic displacive phase transition at $T_{\mathrm{OO}}$.

\section{Second structural phase transition}

Anomalous changes in the lattice parameters were reported to occur on cooling below $T_{\mathrm{s}}$, where $a$ and $c$ increased, accompanied by a decrease in the monoclinic axis, $b$, and an increase in the monoclinic angle, $\beta^{14}$. Together, these changes are related to negative thermal expansion below $T_{\mathrm{s}}$. The same behaviour was observed for our sample, as shown in Figure 1. In the published study $^{14}$, no clear evidence for a deviation from the $I 2 / \mathrm{m}$ crystal structure below $T_{\mathrm{s}}$ had been found, and the physical origin of this phase transition had not been determined.

The $I 2 / m$ crystal structure of our $\mathrm{YMn}_{7} \mathrm{O}_{12}$ sample was refined against neutron powder diffraction data collected above $T_{\mathrm{s}}$, as shown in Figure $3 \mathrm{a}$. An excellent fit to the data was achieved, and the refined structural parameters are given in Table II. The cation site occupancies were fixed to one whilst $\mathrm{Mn}^{2+}$ was free to substitute $\mathrm{Y}^{3+}$, and the average oxygen occupation was freely refined. Within these constraints we determined a sam- 


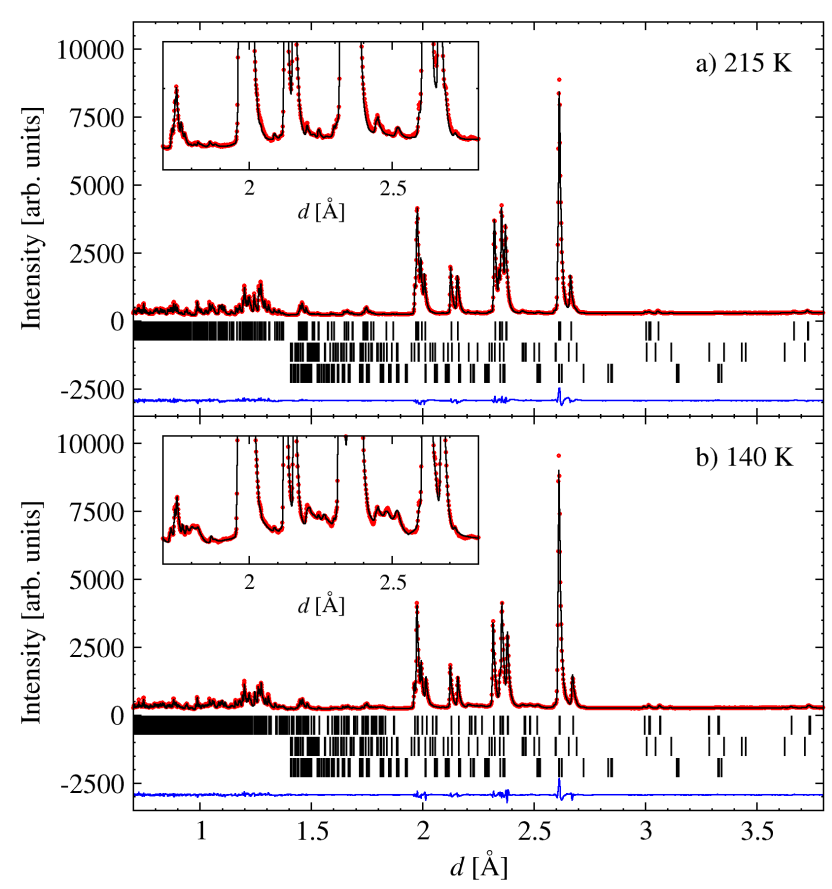

FIG. 3. Neutron powder diffraction data measured from $\mathrm{YMn}_{7} \mathrm{O}_{12}$ in the high resolution back scattering detector bank (average $2 \theta=152^{\circ}$ ) of the WISH diffractometer. Diffraction data (red points) collected above and below $T_{\mathrm{s}}$ are shown in panes a) and b), respectively. The fitted nuclear (top tick marks), and $\mathrm{YMn}_{3} \mathrm{O}_{6}$ and $\mathrm{Mn}_{2} \mathrm{O}_{3}$ impurity (middle and bottom tick marks, cut off below $1.4 \AA$ ) structural models are shown as a solid black line. A difference pattern $\left(I_{\text {obs }}-I_{\text {calc }}\right)$ is given as a blue solid line at the bottom of each pane. The insets to both panes highlight weak diffraction peaks that appear below the structural phase transition at $T_{\mathrm{s}}$.

ple composition of $\mathrm{Y}_{0.8} \mathrm{Mn}_{7.2} \mathrm{O}_{11.6}$. We note that the oxygen content was found to be lower than expected despite heterovalent substitution of manganese for yttrium, and should be taken with caution. Several weak diffraction peaks that could not be indexed using the $\mathrm{YMn}_{7} \mathrm{O}_{12}$ unit cell metric were found to originate in the $\mathrm{YMn}_{3} \mathrm{O}_{6}(\sim 3.1(1)$ wt.\%) impurity, as well as a small $\mathrm{Mn}_{2} \mathrm{O}_{3}(\sim 0.54(6)$ wt.\%) impurity not seen in our synchrotron x-ray powder diffraction data discussed above (because a small sample was taken from a different synthesis batch for the synchrotron x-ray diffraction experiments).

Below $T_{\mathrm{s}}$, small peaks appeared in the neutron powder diffraction data highlighted in the inset of Figure 3b, which were not observed in synchrotron $\mathrm{x}$-ray powder diffraction data collected at $113 \mathrm{~K}$, and hence likely to originate in displacements of oxygen. These additional peaks could be indexed using the $I 2 / \mathrm{m}$ unit cell metric, but they corresponded to reciprocal lattice points forbidden by $I$ centring translational symmetry. Hence, the phase transition at $T_{\mathrm{s}}$ was not isostructural, and the respective symmetry breaking modes had the commensurate propagation vector $\mathbf{k}=(0,1,0)$ - a high symme- try point of the $I 2 / \mathrm{m}$ Brillouin zone. Symmetry analysis was performed using the ISOTROPY suite, taking the refined $I 2 / m$ crystal structure as the parent, and under the assumption that the phase transition involved atomic displacements. Four irreducible representations, $\mathrm{Y}_{1}^{+}, \mathrm{Y}_{2}^{+}, \mathrm{Y}_{1}^{-}$, and $\mathrm{Y}_{2}^{-}$, enter into the decomposition of the Y-point $(\mathbf{k}=(0,1,0))$ reducible representation for the Wyckoff positions of all atomic species. The symmetry adapted displacive modes of the above four irreducible representations lower the crystal symmetry to $P 2 / m$, $P 2_{1} / n, P 2 / n$, and $P 2_{1} / m$, respectively, whilst maintaining the same unit cell metric. Systematic testing of all displacive modes against the neutron powder diffraction data demonstrated that $\mathrm{Y}_{2}^{+}$modes represented a unique model solution. The crystal structure was parameterised within the corresponding subgroup (space group $P 2_{1} / n$, same basis as the $I 2 / m$ unit cell) and refined against the diffraction data (Figure 3b). A good fit to the data was achieved, and the refined crystallographic parameters are given in Table II. We note that the $P 2_{1} / n$ space group assignment below $T_{\mathrm{S}}$ is further supported by the absence of $0 k 0: k$ odd, and $h 0 l: h+l$ odd reflections at all temperatures, which are extinct by the $2_{1}$ and $n$ symmetries, respectively.

The multiplicities of all cation Wyckoff positions remain the same below $T_{\mathrm{s}}$, but the A site point symmetries are lowered from $2 / m$ to $\overline{1}$, and the B site point symmetries are lowered from $\overline{1}$ to 1 . Consequently, the displacement of $\mathrm{B}$ site $\mathrm{Mn}$ ions becomes allowed in a general direction. The $\mathrm{O} 1$ and $\mathrm{O} 2 \mathrm{Wyckoff}$ positions also maintain the same multiplicity through the phase transition, but their site symmetry is lowered from $m$ to 1 , allowing additional atomic displacements along the monoclinic $b$ axis. The $\mathrm{O} 3$ and $\mathrm{O} 4$ anions already adopt general positions in the high temperature phase, but their symmetry equivalent sites undergo a two-fold splitting below $T_{\mathrm{s}}$ as a result of broken translational symmetry. All symmetry breaking atomic displacements occuring below $T_{\mathrm{s}}$ are equal and opposite for atoms related by $I$ centring translational symmetry above $T_{\mathrm{s}}$.

Previous studies of the $\mathrm{YMn}_{7} \mathrm{O}_{12}$ crystal structure, based entirely on the $I 2 / \mathrm{m}$ model, reported an anomalously large isotropic atomic displacement parameter of yttrium below $T_{\mathrm{s}}$, which indicated that its displacement might be associated with the phase transition ${ }^{14}$. However, the $P 2_{1} / n$ subgroup identified above does not support any such displacements. Combined with the $\mathrm{Y}_{2}^{+}$ modes, atomic displacements of yttrium would result in a lowering of the crystal symmetry from $I 2 / m$ to $P c, P 2_{1}$, or $P 1$, which is inconsistent with the observed continuous nature of the phase transition, and also inconsistent with the extinctions observed in our neutron diffraction data. Yet, we also found an anomalously large isotropic atomic displacement parameter of yttrium in our $P 2_{1} / n$ structural refinement below $T_{\mathrm{s}}$ (anomalous behaviour of the yttrium atomic displacement parameter was also found in fits to synchrotron x-ray diffraction data, not shown here). This unphysical result could be reconciled by in- 
TABLE II. Crystal structure parameters of $\mathrm{YMn}_{7} \mathrm{O}_{12}(Z=2)$ refined above and below the structural phase transition at $T_{\mathrm{s}}$. A site manganese fractional coordinates for both phases are Mn1: $[0,0.5,0], \operatorname{Mn} 2:[0.5,0,0]$, and Mn3: $[0.5,0.5,0]$.

\begin{tabular}{|c|c|c|}
\hline & $T>T_{\mathrm{s}}$ & $T<T_{\mathrm{s}}$ \\
\hline SG & $I 2 / m$ & $P 2_{1} / n$ \\
\hline$T[\mathrm{~K}]$ & 215 & 140 \\
\hline \multicolumn{3}{|c|}{ Lattice parameters } \\
\hline$a[\AA]$ & $7.4504(1)$ & $7.47189(9)$ \\
\hline$b[\AA]$ & $7.3261(1)$ & $7.30432(7)$ \\
\hline$c[\AA]$ & $7.4547(1)$ & $7.4634(2)$ \\
\hline$\beta\left[^{\circ}\right]$ & $91.184(1)$ & $91.367(1)$ \\
\hline$V\left[\AA^{3}\right]$ & $406.80(1)$ & $407.21(6)$ \\
\hline
\end{tabular}

\begin{tabular}{|c|c|c|c|c|}
\hline \multirow[t]{3}{*}{$\mathrm{Y}$} & $x$ & 0 & \multicolumn{2}{|c|}{$0.020(4)$} \\
\hline & $y$ & 0 & \multicolumn{2}{|c|}{$-0.008(3)$} \\
\hline & $z$ & 0 & \multicolumn{2}{|c|}{$-0.009(2)$} \\
\hline \multirow[t]{3}{*}{ Mn4 } & $x$ & 0.25 & \multicolumn{2}{|c|}{$0.246(4)$} \\
\hline & $y$ & 0.25 & \multicolumn{2}{|c|}{$0.247(2)$} \\
\hline & $z$ & 0.25 & \multicolumn{2}{|c|}{$0.248(2)$} \\
\hline \multirow[t]{3}{*}{ Mn5 } & $x$ & 0.25 & \multicolumn{2}{|c|}{$0.244(3)$} \\
\hline & $y$ & 0.25 & \multicolumn{2}{|c|}{$0.237(1)$} \\
\hline & $z$ & 0.75 & \multicolumn{2}{|c|}{$0.757(2)$} \\
\hline \multirow[t]{3}{*}{$\mathrm{O} 1$} & $x$ & $0.1618(4)$ & \multicolumn{2}{|c|}{$0.1641(9)$} \\
\hline & $y$ & 0 & \multicolumn{2}{|c|}{$-0.001(1)$} \\
\hline & $z$ & $0.3018(4)$ & \multicolumn{2}{|c|}{$0.3011(5)$} \\
\hline \multirow[t]{3}{*}{$\mathrm{O} 2$} & $x$ & $0.1812(5)$ & \multicolumn{2}{|c|}{$0.182(1)$} \\
\hline & $y$ & 0 & \multicolumn{2}{|c|}{$-0.0098(9)$} \\
\hline & $z$ & $0.6872(5)$ & \multicolumn{2}{|c|}{$0.6861(7)$} \\
\hline \multirow[t]{3}{*}{ O3 } & $x$ & $0.0119(3)$ & $0.009(1)$ & $0.519(1)$ \\
\hline & $y$ & $0.3081(3)$ & $0.3234(8)$ & $0.7960(8)$ \\
\hline & $z$ & $0.1713(3)$ & $0.1877(7)$ & $0.6535(7)$ \\
\hline \multirow[t]{3}{*}{$\mathrm{O} 4$} & $x$ & $0.3110(3)$ & $0.293(1)$ & $0.833(1)$ \\
\hline & $y$ & $0.1738(3)$ & $0.1530(7)$ & $0.6941(7)$ \\
\hline & $z$ & $-0.0103(3)$ & $-0.0049(2)$ & $0.4812(8)$ \\
\hline \multicolumn{5}{|c|}{$\boldsymbol{U}_{\text {iso }}\left[\AA^{2}\right]$} \\
\hline \multicolumn{2}{|c|}{$\mathrm{Y}$} & $0.0101(9)$ & \multicolumn{2}{|c|}{$0.011(1)$} \\
\hline \multicolumn{2}{|c|}{ A site $M n$} & $0.0123(8)$ & \multicolumn{2}{|c|}{$0.0097(8)$} \\
\hline \multicolumn{2}{|c|}{$\mathrm{B}$ site $\mathrm{Mn}$} & $0.0065(6)$ & \multicolumn{2}{|c|}{$0.0067(8)$} \\
\hline \multicolumn{2}{|c|}{$\mathrm{O}$} & $0.0098(3)$ & \multicolumn{2}{|c|}{$0.0090(3)$} \\
\hline
\end{tabular}

Fit reliability parameters

\begin{tabular}{c|c|c}
\hline $\mathrm{R}[\%]$ & 3.23 & 3.27 \\
$w \mathrm{R}[\%]$ & 3.89 & 4.01 \\
$\mathrm{R}_{\text {Bragg }}[\%]$ & 3.07 & 2.85 \\
\hline \hline
\end{tabular}

troducing a small displacement of yttrium, as given in Table II, the sign of which is disordered throughout the crystal hence maintaining $P 2_{1} / n$ symmetry on average. These uncorrelated yttrium displacements away from the average centrosymmetric position generate local dipole moments, forming a dipolar glass-like state.

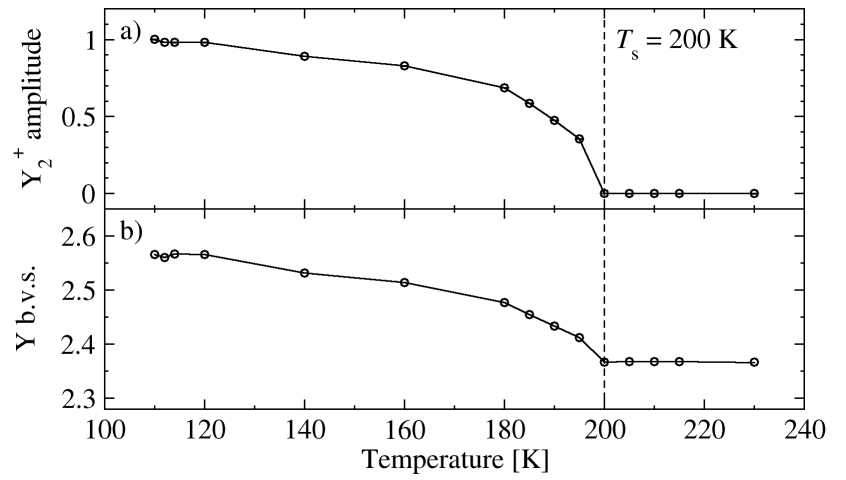

FIG. 4. The temperature dependence of a) the global amplitude of the $\mathrm{Y}_{2}^{+}$modes, and $\mathrm{b}$ ) the $\mathrm{Y}^{3+}$ bond valence sum calculated assuming a fixed $\mathrm{Y}$ displacement away from its average position.

The temperature dependence of the global $\mathrm{Y}_{2}^{+}$amplitude was determined under the assumption that the relative amplitudes of the displacive modes of all atoms remained constant at all temperatures. The result is plotted in Figure 4a, which demonstrates critical behaviour below $T_{\mathrm{s}}$. Relatively little change in $\mathrm{Mn}-\mathrm{O}$ bond lengths was found on cooling below $T_{\mathrm{s}}$, however, Y-O bond lengths varied dramatically as demonstrated by the temperature dependence of the yttrium bond valence sum shown in Figure 4b. The yttrium valence increased from a considerably underbonded value of $\sim 2.4$ above the phase transition, towards the expected value of 3 , below.

Figure 5 graphically illustrates the refined $\mathrm{Y}_{2}^{+}$atomic displacive modes of the six symmetry inequivalent cation - oxygen coordinations, with each pane showing all cations in a single unit cell. The primary distortion of the high temperature $I 2 / m$ crystal structure can be understood as an alternating elongation and contraction

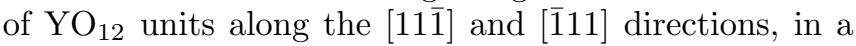
checkerboard pattern within the $[x y \bar{x}]$ plane (Figure $5 f$ ). These distortions are accommodated by a cartwheel rotation of $\mathrm{Mn} 1$ and $\mathrm{Mn} 2 \mathrm{~A}$ site $\mathrm{MnO}_{4}$ square planar units (Figure 5a and b), and a low symmetry distortion of the $\mathrm{B}$ site $\mathrm{MnO}_{6}$ octahedra. The $\mathrm{YO}_{12}[x y \bar{x}]$ checkerboard distortion plane is likely selected by the higher temperature monoclinic distortion where $\beta>90^{\circ}$ (in this setting of the space group) and $[1,0, \overline{1}]>[1,0,1]$. The disordered displacements of yttrium are also shown in Figure 5f, which are aligned with the elongation axis of each $\mathrm{YO}_{12}$ coordination such that the bonding conditions of yttrium are further improved.

Finally in this section, we note that an increase in the valence state of cations incorporated into an oxide crystal lattice typically reduces the unit cell volume. To the contrary, in $\mathrm{YMn}_{7} \mathrm{O}_{12}$ we observe negative thermal expansion below $T_{\mathrm{s}}$, which in this case could occur due to disorder of the yttrium atomic displacements. 


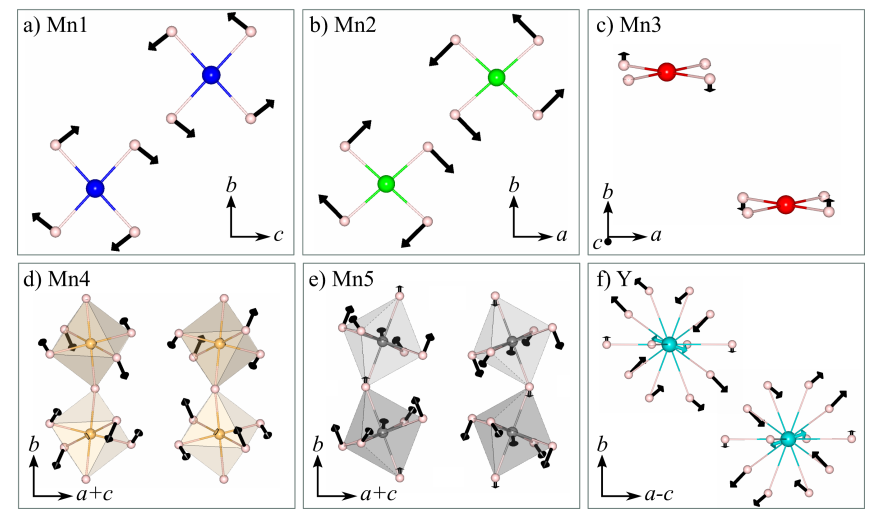

FIG. 5. Selected projections of the $\mathrm{Y}_{2}^{+}$atomic displacive modes (black arrows) of the six symmetry inequivalent cation - oxygen coordinations. All sites within a single unit cell are shown. Mn1, Mn2, Mn3, Mn4, Mn5, and Y are coloured blue, green, red, gold, grey, and cyan, respectively, and oxygen atoms are shown as smaller pink spheres.

\section{Magnetic structure}

The specific heat of $\mathrm{YMn}_{7} \mathrm{O}_{12}$ was measured between 2 and $300 \mathrm{~K}$, and is shown in Figure 6. As previously reported $^{14}$, two anomalies were observed; one at $T_{\mathrm{s}}$, and the other at $\sim 110 \mathrm{~K}$. The temperature dependence of the zero field cooled (ZFC) and field cooled (FC) magnetic susceptibility is also plotted in Figure 6, which demonstrated that the latter anomaly, which we label $T_{1}$, marks a transition from a high temperature paramagnetic phase to a low temperature, long range ordered phase with a net magnetic moment. These data are also consistent with previous reports ${ }^{14}$, and they closely resemble the magnetic susceptibility measured from other $R \mathrm{Mn}_{7} \mathrm{O}_{12}$ compounds ${ }^{16,24}$. However, the magnetic transition temperature of $\mathrm{YMn}_{7} \mathrm{O}_{12}(110 \mathrm{~K})$ is considerably higher than that of the other compounds in this family (La: $79.5 \mathrm{~K}$, Ce: $80 \mathrm{~K}, \mathrm{Nd}: 85 \mathrm{~K}, \mathrm{Sm}: 87 \mathrm{~K}$, and $\mathrm{Eu}: 87 \mathrm{~K})^{16}$. We note the presence of a broad anomaly in the ZFC susceptibility data spanning the approximate temperature range 40 to $70 \mathrm{~K}$, which originated in the $\mathrm{YMn}_{3} \mathrm{O}_{6}$ impurity phase ${ }^{15}$ observed in our diffraction experiments.

Below $T_{1}$, additional magnetic neutron diffraction intensities were observed, which could be unambiguously indexed with the propagation vector $\mathbf{k}=(0,0,0)$, as reported for $\mathrm{YMn}_{7} \mathrm{O}_{12}{ }^{14}$, as well as for other $R \mathrm{Mn}_{7} \mathrm{O}_{12}$ quadruple perovskites ${ }^{16}$. Taking the $P 2_{1} / n$ structure as the parent, the magnetic $\Gamma$-point reducible representation for the relevant Wyckoff positions decomposes into four irreducible representations, $\Gamma_{1}^{+}, \Gamma_{2}^{+}, \Gamma_{1}^{-}$and $\Gamma_{2}^{-}$, corresponding to magnetic space group symmetries of $P 2_{1} / n$, $P 2_{1}^{\prime} / n^{\prime}, P 2_{1} / n^{\prime}$, and $P 2_{1}^{\prime} / n$, respectively ${ }^{22,23}$. The collinear magnetic structure common to other $R \mathrm{Mn}_{7} \mathrm{O}_{12}$ compounds $(R=\mathrm{La}, \mathrm{Ce}, \mathrm{Nd}, \mathrm{Sm}, \mathrm{Eu})^{16}$, which transforms according to the $\Gamma_{2}^{+}$irreducible representation, was refined against the neutron powder diffraction data collected from $\mathrm{YMn}_{7} \mathrm{O}_{12}$ at $1.5 \mathrm{~K}$ (Figure 7 ). An excellent

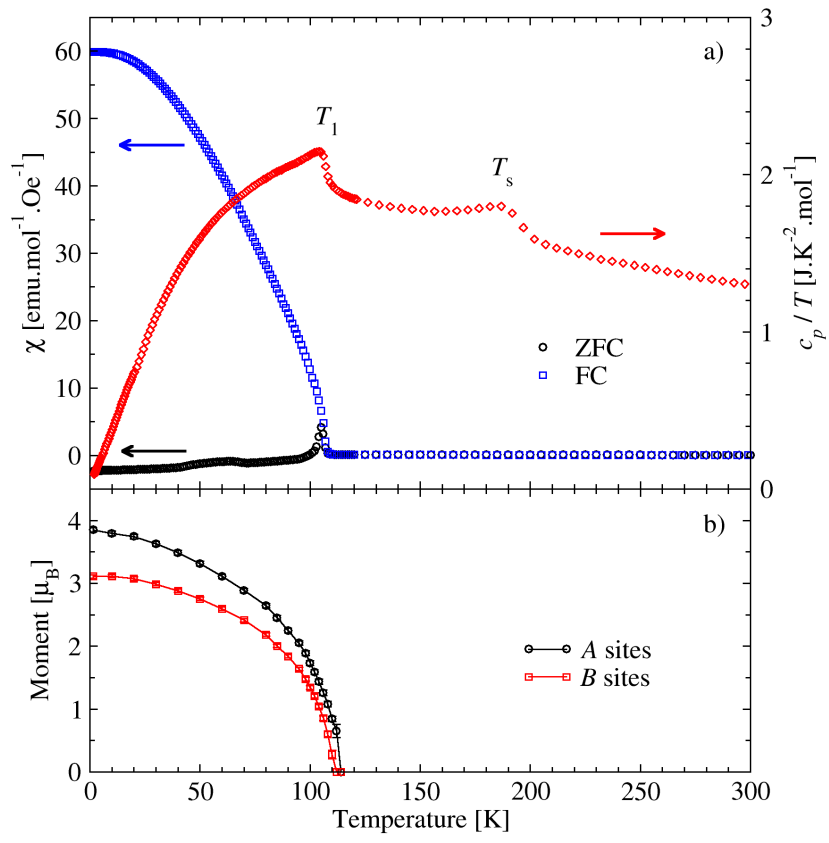

FIG. 6. a) The temperature dependence of the specific heat (red diamonds) measured in zero applied magnetic field, and the zero field cooled (black circles) and field cooled (blue squares) magnetic susceptibility of $\mathrm{YMn}_{7} \mathrm{O}_{12}$. In both cases the magnetic susceptibility was measured using a 100 Oe field. b) The temperature dependence of the ordered magnetic moments.

TABLE III. Magnetic structure parameters of $\mathrm{YMn}_{7} \mathrm{O}_{12}$ refined at $1.5 \mathrm{~K}$. Moment directions are given in spherical coordinates defined such that $m_{z}=m \cos (\theta) \| c, m_{x}=$ $m \sin (\theta) \| a^{*}$, and $m_{y}=0 \| b$. Symmetry equivalent atoms have the same magnetic moment.

\begin{tabular}{c|cc}
\hline \hline Atom & $m\left[\mu_{\mathrm{B}}\right]$ & $\theta\left[^{\circ}\right]$ \\
\hline Mn1 & $3.82(2)$ & $53.1(3)$ \\
Mn2 & $-3.82(2)$ & $53.1(3)$ \\
Mn3 & $3.82(2)$ & $53.1(3)$ \\
Mn4 & $3.09(2)$ & $53.1(3)$ \\
Mn5 & $-3.09(2)$ & $53.1(3)$ \\
\hline \hline
\end{tabular}

fit to the data was achieved with reliability parameters of $R_{\mathrm{mag}}=3.01 \%, R=3.89 \%$, and $\mathrm{w} R=4.42 \%$, and the refined parameters are given in Table III. Additional antiferromagnetic canting of $\mathrm{B}$ site moments out of the $a c$ plane is allowed within $\Gamma_{2}^{+}$symmetry, however, its introduction into the refinement did not significantly improve the fit. Hence, our data provides no evidence for such a departure from collinearity. Magnetic structure models based upon the other three irreducible representations were exhaustively tested, and were all found to be inconsistent with our diffraction data.

The magnetic structure, depicted in Figure 8, was found to be collinear with all moments lying within the $a c$ 


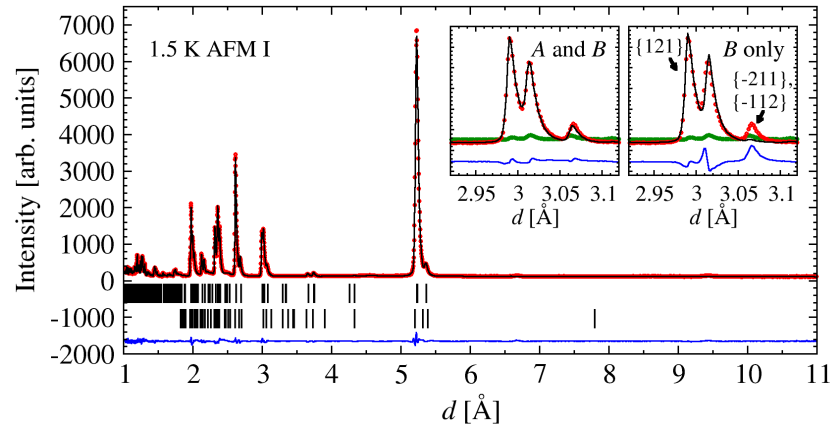

FIG. 7. Neutron powder diffraction data (red points) measured from $\mathrm{YMn}_{7} \mathrm{O}_{12}$ at $1.5 \mathrm{~K}$, in a medium resolution detector bank of the WISH diffractometer with an average $2 \theta=58^{\circ}$. The fitted nuclear+magnetic (top tick marks) and $\sim 2$ wt.\% $\mathrm{YMn}_{3} \mathrm{O}_{6}$ impurity (bottom tick marks, cut off below $1.8 \AA$ ) structural models are shown as a solid black line. A difference pattern $\left(I_{\text {obs }}-I_{\text {calc }}\right)$ is given as a blue solid line at the bottom of each pane. The insets highlight diffraction peaks sensitive to the magnetic ordering of the A site manganese sublattice. Data collected in the paramagnetic phase are shown as green points.

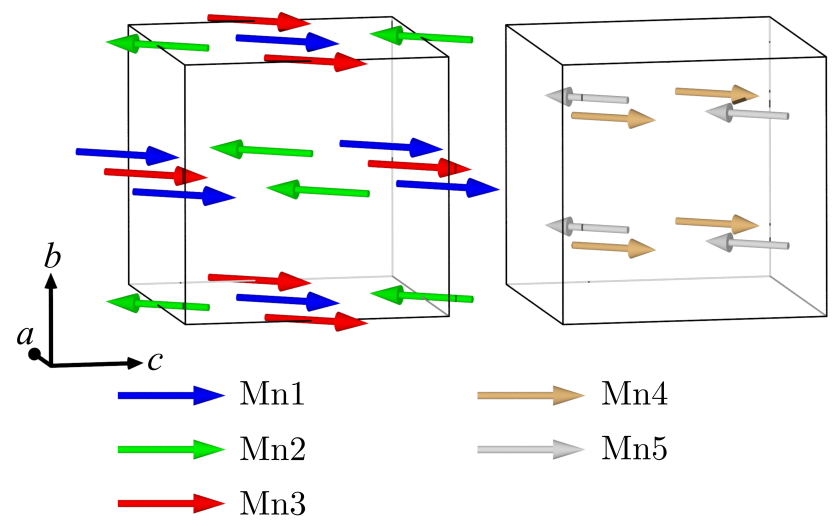

FIG. 8. The refined magnetic structure of $\mathrm{YMn}_{7} \mathrm{O}_{12}$. The $\mathrm{A}$ sublattice is shown on the left, and the B sublattice on the right. The crystallographic unit cell is drawn in solid black lines.

plane. The B site sublattice was antiferromagnetic with parallel Mn4 moments aligned antiparallel to all Mn5 moments. The refinement was found to be insensitive to small differences in the relative magnitude of the moments on the two symmetry inequivalent $B$ sites, which were therefore fixed to be the same. In this case, the collinear B site sublattice carries no net moment. To the contrary, the A site sublattice ordered with a ferrimagnetic structure giving two uncompensated $\mathrm{Mn}^{3+}$ spins per unit cell, or equivalently, $3.8 \mu_{\mathrm{B}}$ per formula unit at $1.5 \mathrm{~K}$ (N.B. to achieve convergence Mn1, Mn2, and Mn3 moments were constrained to have the same magnitude).

The above solution for the $\mathrm{B}$ site magnetic structure is similar to that reported for $\mathrm{YMn}_{7} \mathrm{O}_{12}{ }^{14}$, differing only in its collinearity. However, in the previous work it was

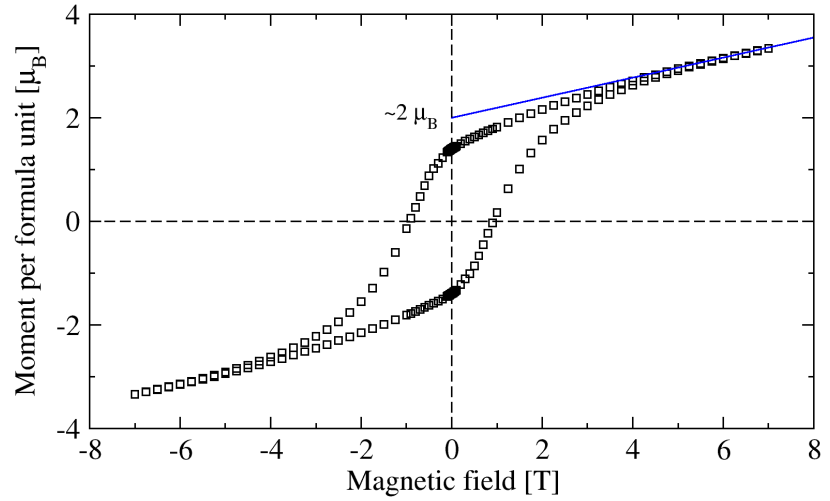

FIG. 9. The magnetic moment per formula unit of our polycrystalline $\mathrm{YMn}_{7} \mathrm{O}_{12}$ sample, measured as a function of magnetic field at $5 \mathrm{~K}$.

found that the A site $\mathrm{Mn}^{3+}$ moments do not order at any temperature. Instead, evidence for latent order was identified in the magnetometry data between 50 and $70 \mathrm{~K}^{14}$. As mentioned above, we observed the same features in the ZFC magnetic susceptibility in this temperature interval, but we can robustly assign this behaviour to the long-range magnetic ordering of the $\mathrm{YMn}_{3} \mathrm{O}_{6}$ impurity phase reported in reference 15. Quantitative evidence for the ordering of the A site $\mathrm{Mn}^{3+}$ moments could be found in our high quality diffraction data, as discussed in detail in reference 16 . In brief, in order to maintain agreement with all magnetic diffraction intensities, the weak magnetic intensity observed at the overlapping $\{\overline{2} 11\}$ and $\{112\}$ peak position could only be accounted for by the refined A site magnetic structure, as highlighted in the inset of Figure 7. The ferrimagnetic ordering of the A site moments is also consistent with bulk magnetometry data shown in Figure 9, in which magnetic hysteresis with a powder averaged moment of $2 \mu_{\mathrm{B}}$ was observed at $5 \mathrm{~K}$. Finally, the temperature dependence of the A and B site magnetic moments is shown in Figure 6, demonstrating concomitant critical behaviour at $T_{1}$.

\section{CONCLUSIONS}

In summary, we have shown that at $640 \mathrm{~K} \mathrm{YMn}_{7} \mathrm{O}_{12}$ undergoes a displacive structural phase transition from a high temperature cubic phase $(\operatorname{Im} \overline{3})$ to a monoclinic, orbitally ordered phase $(I 2 / \mathrm{m})$. The rigid framework of octahedra tilts that primarily accommodate small A site manganese ions leads to an underbonding of yttrium in both $I m \overline{3}$ and $I 2 / m$ structures, which on further cooling results in a second, low temperature structural phase transition at $200 \mathrm{~K}$. This unusual transition is characterised by static atomic displacements, as well as a dipolar-glass-like state of yttrium that can lead to the negative thermal expansion observed below the transition temperature. Both order parameters improve the bond- 
ing conditions of yttrium and in principle can be decoupled. This implies that the correlated and uncorrelated atomic displacements can independently drive low temperature structural phase transitions in other $R \mathrm{Mn}_{7} \mathrm{O}_{12}$ compounds with small rare-earth radius, for example $\mathrm{HoMn}_{7} \mathrm{O}_{12}$ and $\mathrm{ErMn}_{7} \mathrm{O}_{12}{ }^{15}$. We note that the underbonding of $\mathrm{A}$ site cations in a perovskite lattice typically results in structural modifications. However, it is less common to find such structural instabilities driving low temperature phase transitions. The non-perovskite phase, $R \mathrm{BaCO}_{4} \mathrm{O}_{7}$, is one of just a few examples ${ }^{25-27}$. Finally, a low temperature ferrimagnetic structure was solved and found to be similar to that reported for other $R \mathrm{Mn}_{7} \mathrm{O}_{12}$ compounds. The magnetic ground state of $\mathrm{YMn}_{7} \mathrm{O}_{12}$ is therefore unmodified by the low temperature structural phase transition, although the magnetic transition temperature is considerably higher than in other $R \mathrm{Mn}_{7} \mathrm{O}_{12}$ materials.

\section{ACKNOWLEDGMENTS}

RDJ acknowledges support from a Royal Society University Research Fellowship. The work performed in Japan was supported in part by JSPS KAKENHI, Grant Numbers JP15K14133 and JP16H04501. The synchrotron radiation experiments were performed at SPring-8 with the approval of NIMS Synchrotron X-ray Station (Proposal Number: 2018A4501).
* roger.johnson@physics.ox.ac.uk

1 O. Muller and R. Roy., Crystal Chemistry of Non-Metallic Materials (1974).

2 T. Kimura, T. Goto, H. Shintani, K. Ishizaka, T. Arima, and Y. Tokura, Nature 426, 55 (2003).

3 N. Hur, S. Park, P. A. Sharma, J. S. Ahn, S. Guha, and S.-W. Cheong, Nature 429, 392 (2004).

4 E. Dagotto, T. Hotta, and A. Moreo, Physics Reports 344, 1 (2001).

${ }^{5}$ Y. Tokura, Reports on Progress in Physics 69, 797 (2006).

6 A. N. Vasilev and O. S. Volkova, Low Temperature Physics 33, 895 (2007).

7 E. Gilioli and L. Ehm, IUCrJ 1, 590 (2014).

8 R. D. Johnson, F. Mezzadri, P. Manuel, D. D. Khalyavin, E. Gilioli, and P. G. Radaelli, Phys. Rev. Lett. 120, 257202 (2018).

9 P. M. Woodward, Acta Crystallographica Section B 53, 44 (1997).

${ }^{10}$ K. Uusi-Esko, J. Malm, N. Imamura, H. Yamauchi, and M. Karppinen, Materials Chemistry and Physics 112, 1029 (2008).

11 S. Geller, The Journal of Chemical Physics 24, 1236 (1956).

12 M. Marezio, P. Dernier, J. Chenavas, and J. Joubert, Journal of Solid State Chemistry 6, 16 (1973).

13 H. Okamoto, M. Karppinen, H. Yamauchi, and H. Fjellvg, Solid State Sciences 11, 1211 (2009).

14 M. Verseils, F. Mezzadri, D. Delmonte, B. Baptiste, Y. Klein, S. Shcheka, L. C. Chapon, T. Hansen, E. Gilioli, and A. Gauzzi, Phys. Rev. Materials 1, 064407 (2017).

15 L. Zhang, Y. Matsushita, K. Yamaura, and A. A. Belik, Inorg. Chem. 56, 5210 (2017).
${ }^{16}$ R. D. Johnson, D. D. Khalyavin, P. Manuel, L. Zhang, K. Yamaura, and A. A. Belik, Phys. Rev. B 98, 104423 (2018).

17 F. Izumi and T. Ikeda, Mater. Sci. Forum 321-324, 198 (2000).

18 M. Tanaka, Y. Katsuya, and A. Yamamoto, Rev. Sci. Instrum. 79, 075106 (2008).

19 M. Tanaka, Y. Katsuya, Y. Matsushita, and O. Sakata, J. Ceram. Soc. Jpn. 121, 287 (2013).

20 L. C. Chapon, P. Manuel, P. G. Radaelli, C. Benson, L. Perrott, S. Ansell, N. J. Rhodes, D. Raspino, D. Duxbury, E. Spill, and J. Norris, Neutron News 22, $22(2011)$.

21 J. Rodríguez-Carvajal, Physica B 192, 55 (1993).

22 B. J. Campbell, H. T. Stokes, D. E. Tanner, and D. M. Hatch, J. Appl. Crystallogr. 39, 607 (2006).

${ }^{23}$ H. T. Stokes, D. M. Hatch, and B. J. Campbell, "Isotropy," (2007).

24 L. Zhang, N. Terada, R. D. Johnson, D. D. Khalyavin, P. Manuel, Y. Katsuya, M. Tanaka, Y. Matsushita, K. Yamaura, and A. A. Belik, Inorganic Chemistry 57, 5987 (2018).

25 D. D. Khalyavin, L. C. Chapon, P. G. Radaelli, H. Zheng, and J. F. Mitchell, Phys. Rev. B 80, 144107 (2009).

26 M. Avdeev, V. Kharton, and E. Tsipis, Journal of Solid State Chemistry 183, 2506 (2010).

27 D. D. Khalyavin, P. Manuel, B. Ouladdiaf, A. Huq, P. W. Stephens, H. Zheng, J. F. Mitchell, and L. C. Chapon, Phys. Rev. B 83, 094412 (2011). 